\title{
Erratum: Effective field theory after a new-physics discovery
}

\section{Stefan Alte, ${ }^{a}$ Matthias König $^{b}$ and Matthias Neubert ${ }^{a, c}$}

${ }^{a}$ PRISMA Cluster of Excellence $\&$ Mainz Institute for Theoretical Physics, Johannes Gutenberg University, 55099 Mainz, Germany

${ }^{b}$ Physik-Institut, Universität Zürich, CH-8057, Switzerland

${ }^{c}$ Department of Physics $\&$ LEPP, Cornell University, Ithaca, NY 14853, U.S.A.

E-mail: stalte@uni-mainz.de, matthias.koenig@uzh.ch, matthias.neubert@uni-mainz.de

ERRATUM TO: JHEP08(2018)095

ARXiv EPrint: 1806.01278 
The two linear relations between operators shown in eq. (3.29) were missing an integral over the momentum fraction $u$ on the right-hand side. The correct relations are

$$
\begin{aligned}
S & \bar{X}_{L, n_{1}}^{i} i \not \partial_{\perp} x_{L, n_{2}}^{j}+\left(n_{1} \leftrightarrow n_{2}\right) \\
& =\int_{0}^{1} d u\left[\left(\boldsymbol{Y}_{f}\right)^{j k}\left(O_{F_{L} \bar{f}_{R}}^{i k}+O_{F_{L} \bar{f}_{R} \phi}^{(2) i k}\right)-\sum_{r}\left(O_{F_{L} \bar{F}_{L} A_{r}}^{j i}\right)^{\dagger}\right], \\
S & {\left[\bar{X}_{L, n_{1}}^{i}\left(-i \overleftarrow{\phi}_{\perp}\right) x_{L, n_{2}}^{j}\right]+\left(n_{1} \leftrightarrow n_{2}\right) } \\
& =\int_{0}^{1} d u\left[\left(\boldsymbol{Y}_{f}^{*}\right)^{i k}\left[\left(O_{F_{L} \bar{f}_{R}}^{j k}\right)^{\dagger}+\left(O_{F_{L} \bar{f}_{R} \phi}^{(2) j k}\right)^{\dagger}\right]-\sum_{r} O_{F_{L} \bar{F}_{L} A_{r}}^{i j}\right] .
\end{aligned}
$$

In the one-particle anomalous dimensions in eq. (5.7) two fractions were mistyped. The correct expressions read

$$
\begin{aligned}
\gamma^{f_{L}}=\gamma^{\bar{f}_{L}} & =-Y_{f_{L}}^{2} \frac{\alpha_{1}}{4 \pi}-\frac{9 \alpha_{2}}{16 \pi}-\delta_{f q} \frac{\alpha_{3}}{\pi}+\frac{1}{32 \pi^{2}} \boldsymbol{Y}_{f} \boldsymbol{Y}_{f}^{\dagger}, \\
\gamma^{f_{R}}=\gamma^{\bar{f}_{R}} & =-Y_{f_{R}}^{2} \frac{\alpha_{1}}{4 \pi}-\delta_{f q} \frac{\alpha_{3}}{\pi}+\frac{1}{16 \pi^{2}} \boldsymbol{Y}_{f}^{\dagger} \boldsymbol{Y}_{f}, \\
\gamma^{\phi} & =-\frac{\alpha_{1}}{4 \pi}-\frac{3 \alpha_{2}}{4 \pi}+\sum_{f} \frac{N_{c}^{f} y_{f}^{2}}{16 \pi^{2}}
\end{aligned}
$$

In the calculation of the anomalous dimension $\Gamma_{\text {mix }}(u, w, \mu)$ in eq. (5.16) we missed the contribution of a one-particle reducible diagram. When this is included, we find

$$
\Gamma_{\operatorname{mix}}(u, w, \mu)=\frac{C_{F} \alpha_{s}(\mu)}{\pi}\left[\frac{\theta(1-u-w)}{1-u}-(1-w)\right]+\mathcal{O}\left(\alpha_{s}^{2}\right) .
$$

We are grateful to Martin Beneke and Robert Szafron for pointing out this omission.

Open Access. This article is distributed under the terms of the Creative Commons Attribution License (CC-BY 4.0), which permits any use, distribution and reproduction in any medium, provided the original author(s) and source are credited. 